\title{
Detection of Leukocortical Lesions in Multiple Sclerosis and Their Association with Physical and Cognitive Impairment: A Comparison of Conventional and Synthetic Phase-Sensitive Inversion Recovery MRI
}

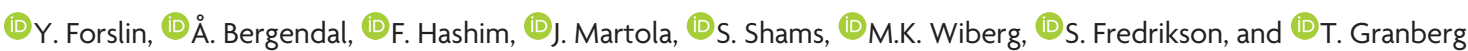

\begin{abstract}
BACKGROUND AND PURPOSE: Cortical lesions are common in multiple sclerosis and are included in the latest diagnostic criteria. The limited sensitivity of cortical MS lesions on conventional MR imaging can be improved by phase-sensitive inversion recovery. Synthetic MR imaging could provide phase-sensitive inversion recovery without additional scanning, but the use of synthetic phase-sensitive inversion recovery remains to be validated. We aimed to compare the ability and clinical value of detecting leukocortical lesions with conventional and synthetic phase-sensitive inversion recovery in MS.
\end{abstract}

MATERIALS AND METHODS: Twenty-one patients with MS prospectively underwent conventional and synthetic phase-sensitive inversion recovery, 3D T1-weighted, and T2 FLAIR imaging. Two neuroradiologists independently performed blinded phase-sensitive inversion recovery lesion assessments; a consensus rating with all sequences was considered the criterion standard. Lesion volumes were segmented. All participants underwent standardized cognitive and physical examinations and Fatigue Severity Scale assessment. Results were analyzed with multiple linear regressions.

RESULTS: Interrater and criterion standard agreement for leukocortical lesions was excellent for both conventional and synthetic phase-sensitive inversion recovery (intraclass correlation coefficient $=0.79-0.97$ ). Leukocortical lesion volumes for both sequences were associated with lower information-processing speed $(P \leq .01)$ and verbal fluency $(P \leq .02)$. Both phase-sensitive inversion recovery sequences showed a positive effect on the association when combining volumes of leukocortical lesions and white matter lesions with information-processing speed $(P \leq .005)$ and verbal fluency $(P \leq .03)$. No associations were found between leukocortical lesion volumes and physical disability or fatigue.

CONCLUSIONS: Synthetic and conventional phase-sensitive inversion recovery have a sensitivity similar to that of leukocortical MS lesions. The detected leukocortical lesions are associated with cognitive dysfunction and thus provide clinically relevant information, which encourages assessment of cortical MS involvement at conventional field strengths.

ABBREVIATIONS: DIR = double inversion recovery; EDSS = Expanded Disability Status Scale; $I C C=$ intraclass correlation coefficient; $L C L=$ leukocortical lesions; $P S I R=$ phase-sensitive inversion recovery; $R_{1}=$ longitudinal relaxation rate; $R^{2}=$ transverse relaxation rate

M ultiple sclerosis is a chronic inflammatory and degenerative disease affecting the central nervous system and is the leading nontraumatic cause of neurologic disability in young adults. ${ }^{1}$

\footnotetext{
Received May 22, 2018; accepted after revision August 2.

From the Departments of Clinical Science, Intervention and Technology (Y.F., A.B. F.H., J.M., S.S., M.K.W., T.G.) and Clinical Neuroscience (S.F.), Karolinska Institutet, Stockholm, Sweden; and Departments of Radiology (Y.F., F.H., J.M., S.S., M.K.W., T.G.) and Neurology (S.F.), Karolinska University Hospital, Stockholm, Sweden. This work was supported by Karolinska Institutet and Stockholm County Council through an ALF grant.

Please address correspondence to Yngve Forslin, MD, Department of Clinical Neuroscience, C1-46, Karolinska University Hospital, 14186 Stockholm, Sweden; e-mail: yngve.forslin@ki.se

- Indicates open access to non-subscribers at www.ajnr.org

7 Indicates article with supplemental on-line photo.

http://dx.doi.org/10.3174/ajnr.A5815
}

In recent years, there has been an increased awareness of the gray matter involvement in MS. Cortical MS lesions are closely associated with cognitive impairment ${ }^{2,3}$ and contribute to cognitive deficits independent of white matter lesions. ${ }^{4-6}$ Cortical lesions are also an independent predictor of conversion from clinically isolated syndrome to MS. ${ }^{7}$ Thus, there is a need for feasible imaging techniques that can also monitor disease evolution and treatment response in the cerebral gray matter.,

While MS lesions in white matter are readily visualized with MR imaging, conventional MR imaging techniques have a low sensitivity for the detection of gray matter MS pathology, which hinders accurate assessment of the total lesion burden. ${ }^{5}$ Newer MR imaging sequences such as double inversion recovery (DIR) and phase-sensitive inversion recovery (PSIR) are 1.5-5 times more sensitive than conventional MR imaging sequences in the 
detection of cortical lesions. ${ }^{4}$ Leukocortical lesions (LCL) are located at the interface between the white matter and the cortex. LCL have the highest detection rate among cortical lesions and are thus a feasible potential imaging biomarker for cognitive deficits that could be readily available for clinical practice. ${ }^{10,11}$

Synthetic MR imaging is a time-efficient MR imaging technique that provides simultaneous quantitative measurements of the longitudinal relaxation rate $\left(\mathrm{R}_{1}\right)$, the transverse relaxation rate $\left(\mathrm{R}_{2}\right)$, and proton-density with correction for field inhomogeneities. ${ }^{12}$ The technique is based on a double-echo saturation-recovery turbo spin-echo sequence applied with 4 repetitions in which the slice acquisition order is changed for each repetition. In practice, this provides 2 different TEs and 4 different TIs for each voxel. Both the magnitude and phase data are saved, providing a total of 16 complex images that are used to fit the T1- and T2-relaxation curves with a computationally efficient least-squares approach. From this simultaneous relaxometry, synthetic MR imaging can provide synthesized images with a wide range of TEs, TRs, and TIs. Thus, it is possible to obtain multiple spin-echo MR imaging weightings from a single acquisition. The technique has been shown to provide proton-density-, T1-, and T2-weighted images with diagnostic quality in MS. ${ }^{13-15}$ Furthermore, it is also possible to synthesize images with inversion pulses and by specifying the TRs, TEs, and TIs as a T1-weighted inversion recovery with phase-sensitive reconstruction; the technique makes it possible to obtain PSIR images from the same sequence without additional scanning time. ${ }^{14}$ This feature makes the technique attractive to apply in the monitoring of pathologies such as MS, in which the detection of cortical lesions on PSIR may be especially clinically important.

We aimed to compare the sensitivity of conventional and synthetic PSIR in detecting leukocortical MS lesions and to evaluate its clinical value in terms of their associations with clinical disability. We hypothesized that synthetic PSIR would have a sensitivity comparable with that of conventional PSIR and that the volume of LCL detected with synthetic PSIR would correlate with cognitive and physical disability.

\section{MATERIALS AND METHODS Study Population}

We prospectively recruited a sample of 21 patients at the MS outpatient clinic at the Department of Neurology, Karolinska University Hospital in Huddinge, Stockholm, Sweden. The inclusion criterion was a diagnosis of MS according to the concurrent diagnostic criteria, ${ }^{16}$ and the exclusion criteria were contraindications for MR imaging, neurologic comorbidities, or a history of head trauma. The cohort was representative of the MS population in our region, represented by all clinical subtypes: 13 relapsing-remitting, 7 secondary-progressive, and 1 primary-progressive. ${ }^{17}$ The demography of the study population is further detailed in Table 1.

\section{Image Acquisition}

All participants were scanned on the same Magnetom Trio 3T MR imaging scanner (Siemens, Erlangen, Germany) using a 12-channel head coil. The imaging protocol included a multidynamic multiecho turbo spin-echo sequence for synthetic MR imaging, conventional PSIR images, and additionally a 3D T1-weighted MPRAGE and T2weighted FLAIR images. Synthetic PSIR images were achieved by
Table 1: Demography of the study population ${ }^{a}$

\begin{tabular}{lc}
\hline & $\begin{array}{c}\text { Patients } \\
\text { with MS }\end{array}$ \\
\hline Female/male & $14: 7$ \\
Age (yr) & $44.5 \pm 12$ \\
Disease duration (yr) & $14.5 \pm 9.7$ \\
MS subtype (RR/SP/PP) (No.) & $13 / 7 / 1$ \\
Disease-modifying therapy (No.) (\%) & $14(67 \%)$ \\
EDSS score (median) (interquartile range) & $2.0(2.0)$ \\
Symbol Digit Modalities Test, $z$ scores (median) & $-0.48(1.46)$ \\
$\quad$ (interquartile range) & $-0.37 \pm 1.37$ \\
Verbal Fluency Test $z$ scores & $4.53 \pm 1.79$ \\
Fatigue Severity Scale score & \\
\hline Note:-RR indicates relapsing-remitting; SP, $\quad$ secondary-progressive; PP, \\
primary-progressive. \\
a Values reported as mean \pm SD unless otherwise specified.
\end{tabular}

applying a phase-sensitive reconstruction on the $\mathrm{T} 1$ inversion recovery parameters from synthetic MR imaging as specified in Table 2. The PSIR reconstruction is performed instantaneously in the synthetic MR imaging software after reading the DICOM images and fitting the quantitative maps (which takes $<20$ seconds on a standard workstation). All acquisition parameters are detailed in Table 2. None of the sequences were acquired with motion correction to accurately reflect clinical image acquisitions.

\section{Radiologic Evaluation}

The radiologic lesion assessments were performed independently by 2 neuroradiologists (F.H. and J.M.), blinded to all clinical information to avoid biased assessments. Using conventional and synthetic PSIR, the neuroradiologists identified juxtacortical lesions and assessed any adjacent cortical involvement, thus reclassifying the lesions as LCL. To compare the performance of conventional and synthetic PSIR and to avoid bias by the influence of other MR imaging sequences, the neuroradiologists initially assessed only these 2 sequences. For each patient, the conventional and synthetic PSIR images were assessed at 2 separate sessions separated by 12 weeks. For half of the participants (randomly assigned), the conventional PSIR image was presented in the first session, and the synthetic PSIR image, in the second session, and vice versa for the other half of the participants. A consensus agreement, considered to be the ground truth, was performed an additional 12 weeks later jointly by the 2 raters. For the consensus rating, both conventional and synthetic PSIR images were available, together with 3D T1-weighted MPRAGE and T2-weighted FLAIR images.

\section{Lesion Segmentations}

WM lesion volumes were segmented on conventional FLAIR images using the lesion probability algorithm in the Lesion Segmentation Toolbox 2.0.12 (Technische Universität München, Munich, Germany) for Statistical Parametric Mapping 12 (SPM12; http://www.fil.ion.ucl.ac.uk/spm/software/spm12). ${ }^{18}$ The resulting WM lesion probability masks were binarized in the FMRIB Software Library 5.0.9 (FSL; (http://www.fmrib.ox.ac.uk/fsl) using a binarization threshold of $0.1{ }^{19} \mathrm{~A}$ resident in radiology (Y.F.) then performed manual corrections of the automatic WM lesion segmentations using ITK-SNAP, Version 3.4.0 (www.itksnap.org).$^{20}$ On the basis of the identified LCL in the consensus agreement assessment, a neuroradiologist (F.H.) manually segmented the LCL in ITK-SNAP on both conventional and synthetic PSIR images separately. 
Table 2: Image-acquisition parameters

\begin{tabular}{lcccc}
\hline \multicolumn{1}{c}{ Sequence Type } & Synthetic PSIR & Conventional PSIR & MPRAGE & FLAIR \\
\hline Acquisition plane & $2 \mathrm{D}$ axial & $2 \mathrm{D}$ axial & 3D sagittal & 3D sagittal \\
Matrix & $256 \times 204$ & $256 \times 204$ & $256 \times 256$ & $256 \times 256$ \\
In-plane resolution $(\mathrm{mm})$ & $0.9 \times 0.9$ & $0.9 \times 0.9$ & $1.0 \times 1.0$ & $1.0 \times 1.0$ \\
Slices (No.) & 34 & 34 & 176 & 1.0 \\
Slice thickness (mm) & 3.0 & 3.0 & - & 1.0 \\
Distance factor & 0.5 & 0.5 & $9^{\circ}$ & - \\
Flip angle & $120^{\circ}$ & $120^{\circ}$ & 2300 & $120^{\circ}, \mathrm{T}^{\circ}$ variable \\
TR (ms) & $4820^{\mathrm{a}}\left(6000^{\mathrm{b}}\right)$ & 6000 & 10 & 6000 \\
TE (ms) & $22 / 100^{\mathrm{a}}\left(10^{\mathrm{b}}\right)$ & 5.98 & 388 \\
TI (ms) & $7: 47$ & $3: 32$ & 900 & 2100 \\
Acquisition time (min:sec) & $150 / 580 / 2000 / 4130^{\mathrm{a}}\left(500^{\mathrm{b}}\right)$ & & $5: 15$ & $7: 02$ \\
\hline
\end{tabular}

Note:- TR indicates repetition time; TE, echo time; TI, inversion time.

a Synthetic MRI is based on a single quantitative acquisition that is then used to generate synthetic images post hoc.

${ }^{\mathrm{b}}$ Settings for the generation of synthetic PSIR are in parentheses.

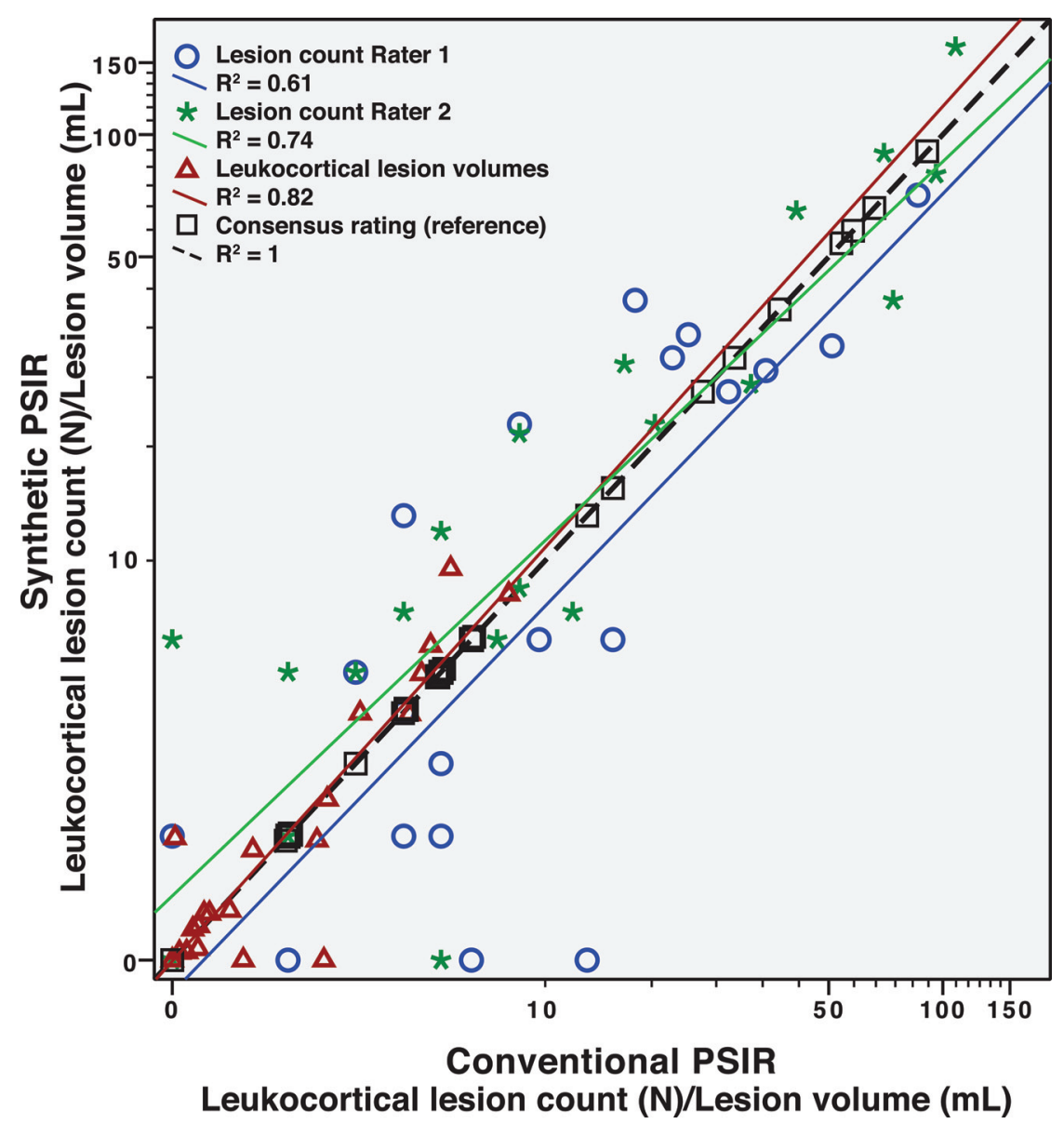

FIG 1. Leukocortical lesion count and volume on conventional and synthetic PSIR. lesion count/volume on conventional and synthetic PSIR images were therefore compared using the Wilcoxon signed rank test. Interrater agreement was evaluated using the intraclass coefficient (ICC); ICC ratings of $<0.40,0.40-$ $0.59,0.60-0.74$, and $0.75-1.0$ were considered weak, fair, good, or excellent according to statistical convention. ${ }^{21}$ Standard multiple linear regression was used to evaluate associations between the EDSS, Fatigue Severity Scale, Symbol Digit Modalities Test, and Verbal Fluency Test $z$ scores (dependent variables) and LCL volume (independent variable). Fatigue and verbal fluency $z$ scores were normally distributed, while Symbol Digit Modalities Test $z$ scores were negatively skewed and therefore underwent a reflect and logarithmic transformation [Lg10 (largest score in data $+1)$ - data] to obtain a normal distribution for the regression analysis; EDSS scores were positively skewed and underwent logarithmic transformation to achieve normal distribution. In a second step, WM lesion volumes were added to the analyses to look for any positive interaction between the 2 lesion metrics. $P<.05$ was considered statistically significant, which after correction for the false discovery rate according to the

\section{Clinical Assessments}

Physical disability was assessed with the Expanded Disability Status Scale (EDSS) by an experienced MS neurologist (S.F.). Cognitive testing was performed by an experienced neuropsychologist (A.B.) with the Symbol Digit Modalities Test, the F-A-S Verbal Fluency Test, and the Fatigue Severity Scale. The testing was performed on the same day as the MR imaging. All cognitive scores were converted into $z$ scores normalized to age and sex.

\section{Statistics}

Normality of data was assessed using the Shapiro-Wilk test. Lesion counts and volumes were positively skewed. Differences in
Benjamini-Hochberg method, corresponded to an adjusted level of $P<.030 .^{22}$

\section{RESULTS}

\section{Lesion Counts and Volumes}

The ICC between the 2 raters was excellent for LCL for both conventional PSIR $(0.79, P<.001)$ and synthetic PSIR $(0.87, P<$ $.001)$. Both raters also had excellent agreement with the consensus rating on both conventional (ICC $=0.91$ and 0.97 , respectively, for each rater, $P<.001$ ) and synthetic PSIR (ICC $=0.92$ and 0.94 , $P<.001)$. There was no significant difference in the number of 
Table 3: Comparison of leukocortical lesion counts and volumes on conventional and synthetic PSIR ${ }^{\mathrm{a}}$

\begin{tabular}{lccccc} 
& $\begin{array}{c}\text { Conventional } \\
\text { PSIR }\end{array}$ & $\begin{array}{c}\text { Synthetic } \\
\text { PSIR }\end{array}$ & $\begin{array}{c}\text { P Value } \\
\text { Conventional vs } \\
\text { Synthetic PSIR }\end{array}$ & $\begin{array}{c}\text { Consensus } \\
\text { Rating }^{\mathbf{b}}\end{array}$ & $\begin{array}{c}\boldsymbol{P} \text { Value Conventional/ } \\
\text { Synthetic PSIR vs } \\
\text { Consensus Rating }\end{array}$ \\
\hline Leukocortical lesion count, Rater 1 (No.) & $7 \pm 17$ & $5 \pm 26$ & .47 & $5 \pm 30$ & $.14 / .06$ \\
Leukocortical lesion count, Rater 2 (No.) & $7 \pm 34$ & $7 \pm 29$ & .08 & $5 \pm 30$ & $.008 / .96$ \\
Leukocortical lesion volume $(\mathrm{mL})$ & $0.53 \pm 2.46$ & $0.32 \pm 2.89$ & .17 & & \\
\hline
\end{tabular}

all values are given as median \pm interquartile range.

${ }^{\mathrm{b}} \mathrm{P}$ value by the Wilcoxon signed rank test.

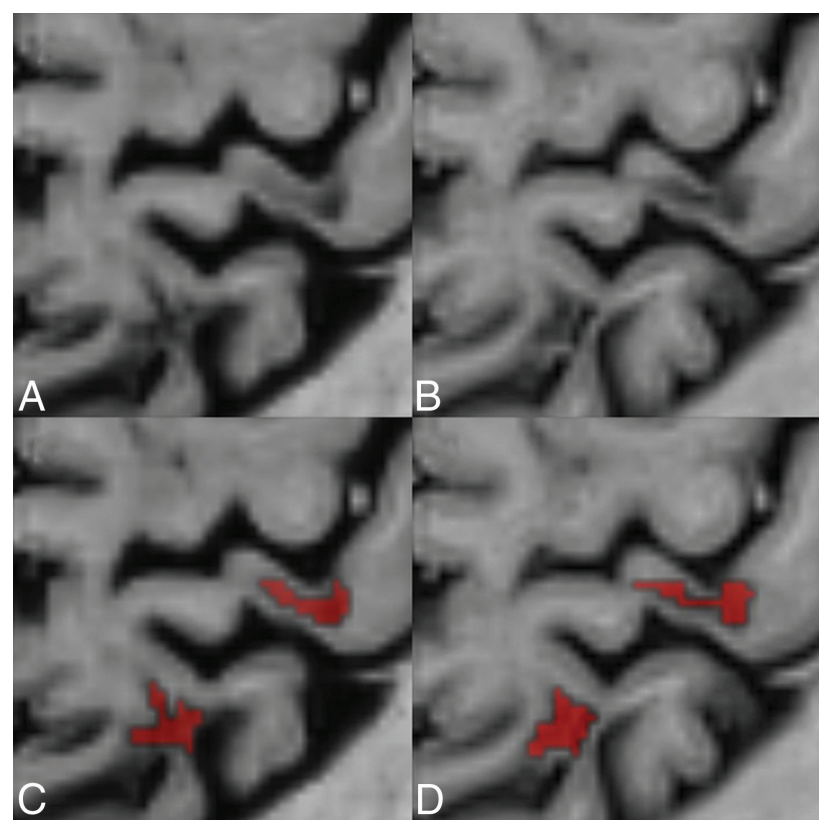

FIG 2. Comparison between conventional and synthetic phase-sensitive inversion recovery. $A$ comparison between conventional $(B$ and $D)$ and synthetic phase-sensitive inversion recovery ( $A$ and $C$ ) illustrates 2 leukocortical MS lesions in a 40-year-old female patient with MS. Lower row illustrates the manual segmentation of the lesions by a neuroradiologist.

detected LCL between conventional and synthetic PSIR $(P=.47$ and $P=.08$, respectively, for each rater). Figure 1 illustrates the relation of the individual lesion ratings and the consensus rating as well as the relation between conventional and synthetic PSIR. The 2 raters seemed to have relatively larger differences in their LCL counts in patients with fewer lesions. When we compared each individual rating with the consensus rating, one of the raters showed a small-but-significant difference between the individual and consensus rating in the LCL count on conventional PSIR ( $P=$ .008 , by the Wilcoxon signed rank test). There was no significant difference between the manually segmented LCL volumes on conventional and synthetic PSIR $(P=.17)$. A detailed comparison of the lesion counts and volumes is presented in Table 3. Figure 2 illustrates the appearance of 2 leukocortical lesions on conventional and synthetic PSIR.

\section{Associations with Dysfunction}

Multiple linear regression showed that higher volumes of LCL were associated with lower Symbol Digit Modalities Test $z$ scores, reflecting information-processing speed, both with measurements from conventional $\left(\beta=-0.62, P=.003\right.$, adjusted $\mathrm{R}^{2}=$ $0.35)$ and synthetic PSIR $\left(\beta=-0.55, P=.010\right.$, adjusted $\mathrm{R}^{2}=$ 0.26). Similarly, higher volumes of LCL on conventional PSIR $\left(\beta=-0.51, P=.019\right.$, adjusted $\left.\mathrm{R}^{2}=0.22\right)$ were associated with lower Verbal Fluency Test $z$ scores, and a similar trend was seen for synthetic PSIR ( $\beta=-0.43, P=.054$, adjusted $\mathrm{R}^{2}=0.14$ ). Using both the LCL and WM lesion volumes from conventional MR imaging, we saw a positive effect on the association for both the Symbol Digit Modalities Test $(\beta=-0.66, P=.001$, adjusted $\left.\mathrm{R}^{2}=0.41\right)$ and the Verbal Fluency Test $(\beta=-0.52, P=.015$, adjusted $\left.\mathrm{R}^{2}=0.24\right)$. An increased association was similarly observed for synthetic PSIR with the Symbol Digit Modalities Test $\left(\beta=-0.58, P=.005\right.$, adjusted $\left.\mathrm{R}^{2}=0.31\right)$ and the Verbal Fluency Test $\left(\beta=-0.47, P=.030\right.$, adjusted $\left.\mathrm{R}^{2}=0.18\right)$.

There were no statistically significant associations between EDSS scores and conventional PSIR $(\beta=0.45, P=.18)$ or synthetic PSIR ( $\beta=0.60, P=.12)$. Neither were there any associations between fatigue and volumes of LCL on conventional PSIR ( $\beta=0.04, P=.88)$ or synthetic PSIR $(\beta=-0.03, P=.89)$.

\section{DISCUSSION}

In this prospective cohort of 21 patients with MS, we show that synthetic PSIR based on the multiparametric synthetic MR imaging technique shows a performance comparable with that of conventional PSIR in detecting leukocortical MS lesions. We further show that larger volumes of LCL on both synthetic and conventional PSIR are associated with lower cognitive performance, thus suggesting that the finding of LCL on PSIR is clinically valuable.

Visualization of cortical pathology in vivo improves the diagnostic accuracy in MS and its differential diagnoses. ${ }^{23}$ A singlecenter study and a larger multicenter study have demonstrated that including cortical lesions in the criteria for dissemination in space in clinically isolated syndrome increases the specificity in the prediction of those who later convert to MS. ${ }^{7,24}$ Our results support the potential clinical feasibility of including the combined term "cortical/juxtacortical lesions" in the evaluation of dissemination in space in the latest MAGNIMS criteria for MS diagnostics and the latest revision of the diagnostic criteria for MS. ${ }^{9,23}$

Including cortical lesions in the diagnostic algorithms for MS has also been previously proposed, ${ }^{7}$ though a histopathologic validation study later showed a fairly low cortical lesion detection rate of merely $18 \%$ with double inversion recovery. ${ }^{25}$ However, PSIR has been suggested to be superior to DIR in detecting cortical MS lesions. ${ }^{26}$ The excellent agreement of LCL on both conventional and synthetic PSIR in the current study indicates that the proposed rating of LCL on PSIR may give a robust assessment of cortical disease involvement in MS. ${ }^{27,28}$ Nevertheless, we found that there was less difference in the LCL count between the 2 different PSIR sequences than between the raters and the consensus rating. This might be because the consensus rating generated a 
larger total lesion burden when the overall sensitivity and specificity increased with the combination of all available sequences. Combining different sequences, as performed for the criterion standard, was subjectively the preferred approach by the raters in the current study to accurately delineate cortical involvement, which supports previously proposed multimodal reading protocol approaches. ${ }^{4,29-31}$

Synthetic MR imaging has previously been shown to provide proton-density-, T1-, and T2-weightings in diagnostic quality (as illustrated in the On-line Figure $)^{13-15}$ as well as automatic volumetrics, ${ }^{13}$ with a single acquisition. The image quality of synthetic FLAIR images has, however, been shown to be hampered by artifacts. ${ }^{13}$ We here show that it is possible to obtain diagnostic synthetic PSIR images from the same acquisition without additional scanning, thus providing a clinically feasible way to visualize leukocortical MS pathology, relevant for the latest revision of the MS criteria. ${ }^{23}$ Nevertheless, if the purpose would be to solely acquire a PSIR contrast, the conventional PSIR would be a faster approach (3 minutes and 32 seconds versus 7 minutes and 47 seconds) but without the additional imaging information provided with synthetic MR imaging.

In terms of the clinical importance of LCL, we found a significant association between higher LCL volume (measured on both synthetic and conventional PSIR) and lower cognitive scores. The good correspondence of both PSIR methods with the cognitive scores is expected because both sequences had similar detection rates and volumes of LCL. This association was increased when adding WM lesion volume to the analyses for both sequences, showing the clinical importance of also detecting LCL with the PSIR methods used here. However, no associations with physical disability or fatigue were found, suggesting that the LCL burden is more related to cognitive disabilities. To further expand our understanding of the pathologic meaning of the imaging findings on conventional/synthetic MR imaging, future studies may investigate the association with biofluid markers of interest in MS.

This study has some limitations: The sample size is relatively small, making it unfeasible to perform additional analyses within the different MS subtypes. The sparse number of raters makes the interrater assessment less robust. Furthermore, a histopathologic validation was not possible in this in vivo study. A comparison with an ultra-high-field strength MR imaging scanner for the ground truth would have been a more optimal validation, but that was, unfortunately, not available for the purpose of the study and synthetic MR imaging has yet to be applied at 7T. A slice distance factor of 0.5 was used to avoid interslice talk. A complementing $3 \mathrm{D}$ acquisition approach, as used for the consensus agreement, could be valuable to further increase the detection of smaller lesions such as purely intracortical lesions. With this in mind, we harmonized the spatial resolutions for conventional and synthetic PSIR so that the comparability of the LCL detection rate was not confounded by partial volume effects.

\section{CONCLUSIONS}

Synthetic MR imaging provides PSIR with a sensitivity similar to that of conventional PSIR in terms of the detection of leukocortical MS lesions. The leukocortical burden detected with synthetic PSIR is associated with cognitive deficits and, therefore, is of clin- ical relevance in MS. Our results highlight the value of evaluating leukocortical MS lesions, even without the use of ultra-high-field scanners and suggest that either synthetic or conventional PSIR could be a part of a multimodal approach with additional 3Dbased sequences, applied to meet the new demands of the latest revision of the MS diagnostic criteria.

\section{ACKNOWLEDGMENTS}

We thank Russell Ouellette for valuable comments on the manuscript.

Disclosures: Yngve Forslin, Åsa Bergendal, Farouk Hashim, Juha Martola, Sara Shams, Tobias Granberg, Maria Kristoffersen-Wiberg—RELATED: Grant: ALF Grant* from Karolinska Institutet and Stockholm City Council. *Money paid to the institution. Sten Fredrikson—UNRELATED: Board Membership: Merck, Sanofi Genzyme, Teva Pharmaceutical Industries, Novartis, Roche; Consultancy: Merck, Sanofi Genzyme, Teva Pharmaceutical Industries, Novartis, Roche; Payment for Lectures Including Service on Speakers Bureaus: Merck, Sanofi Genzyme, Teva Pharmaceutical Industries, Novartis, Roche; Payment for Development of Educational Presentations: Merck, Sanofi Genzyme, Teva Pharmaceutical Industries, Novartis, Roche.

\section{REFERENCES}

1. Peterson JW, Trapp BD. Neuropathobiology of multiple sclerosis. Neurol Clin 2005;23:107-29, vi-vii Medline

2. Chiaravalloti ND, DeLuca J. Cognitive impairment in multiple sclerosis. Lancet Neurol 2008;7:1139-51 CrossRef Medline

3. Odenthal C, Coulthard A. The prognostic utility of MRI in clinically isolated syndrome: a literature review. AJNR Am J Neuroradiol 2015; 36:425-31 CrossRef Medline

4. Nelson F, Poonawalla AH, Hou P, et al. Improved identification of intracortical lesions in multiple sclerosis with phase-sensitive inversion recovery in combination with fast double inversion recovery MR imaging. AJNR Am J Neuroradiol 2007;28:1645-49 CrossRef Medline

5. Geurts JJ, Bö L, Pouwels PJ, et al. Cortical lesions in multiple sclerosis: combined postmortem MR imaging and histopathology. AJNR Am J Neuroradiol 2005;26:572-77 Medline

6. Calabrese M, Agosta F, Rinaldi F, et al. Cortical lesions and atrophy associated with cognitive impairment in relapsing-remitting multiple sclerosis. Arch Neurol 2009;66:1144-50 Medline

7. Filippi M, Rocca MA, Calabrese M, et al. Intracortical lesions: relevance for new MRI diagnostic criteria for multiple sclerosis. $\mathrm{Neu}$ rology 2010;75:1988-94 CrossRef Medline

8. Rocca MA, Amato MP, De Stefano N, et al; MAGNIMS Study Group. Clinical and imaging assessment of cognitive dysfunction in multiple sclerosis. Lancet Neurol 2015;14:302-17 CrossRef Medline

9. Filippi M, Rocca MA, Ciccarelli O, et al; MAGNIMS Study Group. MRI criteria for the diagnosis of multiple sclerosis: MAGNIMS consensus guidelines. Lancet Neurol 2016;15:292-303 CrossRef Medline

10. Nelson F, Datta S, Garcia N, et al. Intracortical lesions by 3T magnetic resonance imaging and correlation with cognitive impairment in multiple sclerosis. Mult Scler 2011;17:1122-29 CrossRef Medline

11. Nielsen AS, Kinkel RP, Madigan N, et al. Contribution of cortical lesion subtypes at 7T MRI to physical and cognitive performance in MS. Neurology 2013;81:641-49 CrossRef Medline

12. Warntjes JB, Leinhard OD, West J, et al. Rapid magnetic resonance quantification on the brain: optimization for clinical usage. Magn Reson Med 2008;60:320-29 CrossRef Medline

13. Granberg T, Uppman M, Hashim F, et al. Clinical feasibility of synthetic MRI in multiple sclerosis: a diagnostic and volumetric validation study. AJNR Am J Neuroradiol 2016;37:1023-29 CrossRef Medline

14. Hagiwara A, Hori M, Yokoyama K, et al. Synthetic MRI in the detection of multiple sclerosis plaques. AJNR Am J Neuroradiol 2017;38: 257-63 CrossRef Medline 
15. Krauss W, Gunnarsson M, Nilsson M, et al. Conventional and synthetic MRI in multiple sclerosis: a comparative study. Eur Radiol 2018;28:1692-1700 CrossRef Medline

16. Polman CH, Reingold SC, Banwell B, et al. Diagnostic criteria for multiple sclerosis: 2010 revisions to the McDonald criteria. Ann Neurol 2011;69:292-302 CrossRef Medline

17. Lublin FD, Reingold SC, Cohen JA, et al. Defining the clinical course of multiple sclerosis: the 2013 revisions. Neurology 2014;83:278-86 CrossRef Medline

18. Schmidt P, Gaser C, Arsic M, et al. An automated tool for detection of FLAIR-hyperintense white-matter lesions in multiple sclerosis. Neuroimage 2012;59:3774-83 CrossRef Medline

19. Smith SM, Jenkinson M, Woolrich MW, et al. Advances in functional and structural MR image analysis and implementation as FSL. Neuroimage 2004;23(Suppl 1):S208-19 CrossRef Medline

20. Yushkevich PA, Piven J, Hazlett HC, et al. User-guided 3D active contour segmentation of anatomical structures: significantly improved efficiency and reliability. Neuroimage 2006;31: 1116-28 CrossRef Medline

21. Cicchetti D. Guidelines, criteria, and rules of thumb for evaluating normed and standardized assessment instrument in psychology. Psychological Assessment 1994;6:284-90 CrossRef

22. Benjamini Y, Hochberg Y. Controlling the false discovery rate: a practical and powerful approach to multiple testing. Journal of the Royal Statistical Society Series B (Methodological) 1995;57:289-300

23. Thompson AJ, Banwell BL, Barkhof F, et al. Diagnosis of multiple sclerosis: 2017 revisions of the McDonald criteria. Lancet Neurol 2018;17:162-73 CrossRef Medline
24. Preziosa P, Rocca MA, Mesaros S, et al. Diagnosis of multiple sclerosis: a multicentre study to compare revised McDonald-2010 and Filippi-2010 criteria. J Neurol Neurosurg Psychiatry 2018;89: 316-18 CrossRef Medline

25. Seewann A, Kooi EJ, Roosendaal SD, et al. Postmortem verification of MS cortical lesion detection with 3D DIR. Neurology 2012;78: 302-08 CrossRef Medline

26. Sethi V, Yousry TA, Muhlert N, et al. Improved detection of cortical MS lesions with phase-sensitive inversion recovery MRI. J Neurol Neurosurg Psychiatry 2012;83:877-82 CrossRef Medline

27. Nielsen AS, Kinkel RP, Tinelli E, et al. Focal cortical lesion detection in multiple sclerosis: 3 Tesla DIR versus 7 Tesla FLASH-T2. J Magn Reson Imaging 2012;35:537-42 CrossRef Medline

28. Mainero $\mathrm{C}$, Benner $\mathrm{T}$, Radding $\mathrm{A}$, et al. In vivo imaging of cortical pathology in multiple sclerosis using ultra-high field MRI. Neurology 2009;73:941-48 CrossRef Medline

29. Favaretto A, Poggiali D, Lazzarotto A, et al. The parallel analysis of phase sensitive inversion recovery (PSIR) and double inversion recovery (DIR) images significantly improves the detection of cortical lesions in multiple sclerosis (MS) since clinical onset. PLoS One 2015;10:e0127805 CrossRef Medline

30. Nelson F, Poonawalla A, Datta S, et al. Is 3D MPRAGE better than the combination DIR/PSIR for cortical lesion detection at 3T MRI? Mult Scler Relat Disord 2014;3:253-57 CrossRef Medline

31. Maranzano J, Rudko DA, Arnold DL, et al. Manual segmentation of MS cortical lesions using MRI: a comparison of 3 MRI reading protocols. AJNR Am J Neuroradiol 2016;37:1623-28 CrossRef Medline 